Pacific Journal of Mathematics

APPROXIMATION BY RATIONAL MODULES ON NOWHERE
DENSE SETS 


\section{APPROXIMATION BY RATIONAL MODULES ON NOWHERE DENSE SETS}

\section{JAMES LI-MING WANG}

Let $X$ be a compact subset of the complex plane. Let the module $\mathscr{R}(X) \overline{\mathscr{P}}_{m}$ be the space of all functions of the form

$$
r_{0}(z)+r_{1}(z) \bar{z}+\cdots+r_{m} \bar{z}^{m}
$$

where each $r_{i}$ is a rational function with poles off $X$. We prove that $\mathscr{R}(X) \overline{\mathscr{P}}_{1}$ is dese in $L^{p}(X)$ for all $1 \leqq p<\infty$ and $\mathscr{R}(X) \overline{\mathscr{P}}_{2}$ is dense in $\mathscr{C}(X)$ if $X$ has no interior point. As corollaries, we also prove that $\mathscr{R}(X) \overline{\mathscr{P}}_{2}$ is dense in lip $(\alpha, X)$ for all $0<\alpha<1$ and $\mathscr{R}(X) \mathscr{\mathscr { P }}_{3}$ is dense in $D^{1}(X)$ for the same $X$.

1. Introductions, Let $X$ be a compact subset of the complex plane. Let the module $\mathscr{R}(X) \overline{\mathscr{S}}_{m}$ be the space $\mathscr{R}+\mathscr{R} \bar{z}+\cdots+\mathscr{R} \bar{z}^{m}$

$$
=\left\{r_{0}(z)+r_{1}(z) \bar{z}+\cdots+r_{m}(z) \bar{z}^{m}\right\},
$$

where each $r_{i}$ is a rational function with poles off $X$. In [3, 4], O'Farrell has studied the relation of the problems of approximation by rational modules in different Lipschitz norms, and in the uniform norm, etc., to one another.

In this note, we investigate the problem of determining the set $X$ so that $\mathscr{R}(X) \overline{\mathscr{P}}_{m}$ is uniformly dense in $\mathscr{C}(X)$ for each $m$.

Vitushkin [8] has given a necessary and sufficient condition in terms of analytic capacities for the case $m=0$. In [3], O'Farrell has given an example of an $X$ such that $\mathscr{R}(X) \overline{\mathscr{P}}_{1}$ is uniformly dense in $\mathscr{C}(X)$ whereas $\mathscr{R}(X)$ fails to be dense in $\mathscr{C}(X)$.

It is apparent that if $X$ has interior, then $\mathscr{R}(X) \overline{\mathscr{P}}_{m}$ can not be dense in $\mathscr{C}(X)$. Thus we restrict our attention to a compact set $X$ without interior throughout this note. Let $L^{p}(X)=L^{p}\left(\chi_{X} d m\right)$, where $d m$ denotes the 2-dimensional Lebesgue measure. We prove the following theorem:

THEOREM. Let $X$ be a compact set with no interior. Then

I. $\mathscr{R}(X) \overline{\mathscr{P}}_{1}$ is dense in $L^{p}(X)$ for all $1 \leqq p<\infty$, and

II. $\mathscr{R}(X) \mathscr{\mathscr { P }}_{2}$ is dense in $\mathscr{C}(X)$.

2. Proof of theorem. Let $\mu$ be a (finite Borel) measure on $X$. The Cauchy transform $\hat{\mu}$ is defined by 


$$
\widehat{\mu}(z)=\int_{x} \frac{d \mu(\zeta)}{\zeta-z} .
$$

It is well known that $\hat{\mu}$ is absolutely convergent for almost all $z(d m)$ and $\hat{\mu}$ belongs to $L_{\text {loc }}^{p}$ for $1 \leqq p<2$ (see [2], p. 37). If $g$ is a function on $X$, we write $\hat{g}$ for $g d m$. Sinanjan [6] was the first to prove that $\mathscr{R}(X)$ is dense in $L^{p}(X)$ when $1 \leqq p<2$. However, it is Brennan's [1] proof that leads to the results we obtain here.

We use the symbol $\bar{\partial}$ for the operator $\partial / \partial x+i(\partial / \partial y)$ and write $g \perp \mathscr{R}(X) \overline{\mathscr{S}}_{m}$ if $\int f g d m=0$ for all $f \in \mathscr{R}(X) \overline{\mathscr{S}}_{m}$. The following lemma is a special case of the key lemma in [3].

Lemma. Let $\mu$ be a measure on $X$. Then $\mu \perp \mathscr{R}(X) \overrightarrow{\mathscr{T}}_{m+1}$ if and only if $\hat{\mu} \perp \mathscr{R}(X) \cdot \overline{\mathscr{P}}_{m}$. [2]).

Proof. Because $\int f d \mu=\pi^{-1} \int(\bar{\partial} f) \hat{\mu} d m$ for all $f \in \mathscr{R}(X) \overline{\mathscr{P}}_{m+1}$ (see

Proof of theorem. Of course, the case $1 \leqq p<2$ is contained in Sinanjan's theorem.

Let $g \in L^{p}(X), q>1, p^{-1}+q^{-1}=1$, such that $g \perp \mathscr{R}(X) . \overline{\mathscr{P}}_{1}$. Lemma implies $\hat{g} \perp \mathscr{R}(X)$. In particular, $\widehat{\hat{g}}=0$ if $z \notin X$. Being the convolution of $g$ and $\zeta^{-1} \in L_{100}^{r}(1 \leqq r<2), \hat{g}$ belongs to $L^{s}(X)$ for some $s>2$, by the classical Young's inequality (see [7], p. 271). And being the convolution of $\hat{g}$ and $\zeta^{-1}, \hat{g}$ is continuous everywhere in the plane. It follows that $\hat{\hat{g}} \equiv 0$ since $X$ has no interior. It is well known that this implies $\hat{g}=0$ in $L^{s}(X)$, which in turn implies $g=0$ in $L^{q}(X)$ and hence $\mathscr{\mathscr { R }}(X) \overline{\mathscr{P}}_{1}$ is dense in $L^{p}(X)$. Part I is proved.

Let $\mu$ be a measure on $X$ such that $\mu \perp \mathscr{R}(X) \cdot \overline{\mathscr{F}}_{2}$. Lemma implies $\hat{\mu} \perp \mathscr{R}(X) \cdot \overline{\mathscr{P}}_{1}$ and Part I implies $\hat{\mu}=0$ and hence $\mu=0$. Part II is proved.

3. Some remarks. Let $\mathscr{E}$ and $\mathscr{D}$ be the usual linear topological space of complex-valued $\mathscr{C}^{\infty}$ functions on the complex plane. Let $\|\cdot\|_{1}$ and $\|\cdot\|_{2}$ be two norms on $\mathscr{D}$ and $\mathscr{E}$ respectively. $\mathscr{R}(X) \mathscr{\mathscr { P }}_{m}$ can be regarded as a subspace of $\left.\mathscr{D}\right|_{X}$ and $\left.\mathscr{E}\right|_{X}$. We denote the closure (i.e., the completion) of $\mathscr{R}(X) \overline{\mathscr{P}}_{m}$ with respect to the norm $\|\cdot\|_{j}$ (restricted to $X$ ) by $\left[\mathscr{R}(X) \ddot{\mathscr{P}}_{m}\right]_{j}$, for $j=1,2$. O'Farrell's theorems in [3] can be roughly stated in the following general form:

If $\phi \rightarrow \hat{\phi}$ is continuous from $\left(\mathscr{D},\|\cdot\|_{1}\right)$ to $\left(\mathscr{E},\|\cdot\|_{2}\right)$, then 
$\left[\mathscr{R}(X) \overline{\mathscr{P}}_{m}\right]_{1}=[\mathscr{D}]_{1}$ implies $\left[\mathscr{R}(X) \overline{\mathscr{P}}_{m+1}\right]_{2}=[\mathscr{E}]_{2}$.

As corollaries, we have the following:

Corollary. Let $X$ be a compact set with no interior. Then

I. $\mathscr{R}(X) \overline{\mathscr{S}}_{2}$ is dense in $\operatorname{lip}(\alpha, X)$ for all $0<\alpha<1$, and

II. $\mathscr{R}(X) \overline{\mathscr{P}}_{3}$ is dense in $D^{1}(X)$.

For the definitions of $\operatorname{lip}(\alpha, X)$ and $D^{1}(X)$, we refer the reader to [3].

It is not known whether $\mathscr{R}(X) \mathscr{\mathscr { P }}_{1}$ is dense in $\mathscr{C}(X)$ for all $X$ without interior. The annular Swiss Cheese of A. Roth [5] has the property that the continuous function $|z|^{2}$ is not in the uniform closure of $\mathscr{R}(X)$. However, it is not clear whether or not the function $|z|$ is in the uniform closure of $\mathscr{R}(X) \overline{\mathscr{P}}_{1}$.

\section{REFERENCES}

1. J. Brennan, Invariant subspaces and rational approximation, J. Functional Analysis, 7 (1971), 285-310.

2. J. Garnett, Analytic capacity and measure, Springer Lecture Notes in Math., No. 297, 1973.

3. A. O'Farrell, Annihilators of rational modules, J. Functional Analysis, 19 (1975), 373-389.

4. - Hausdorff content and rational approximation in fractional Lipschitz norms, Trans. Amer. Math Soc., 228 (1977), 187-206.

5. A. Roth, Approximationseigenschaften und Strahlengrenzwerte meromorpher und ganzer Funktionen, Comment. Math. Helv., 11 (1938), 77-125.

6. S. O. Sinanjan, The uniqueness property of analytic functions on closed sets without interior points, Sibirsk Mat. Z., 6 (1965), 1365-1381.

7. E. M. Stein, Singular Integrals and Differentiability Properties of Functions, Princeton, 1970.

8. A. G. Vitushkin, Analytic capacity of sets and problems in approximation theory, Russian Math. Survey, 22 (1967), 139-200.

Received May 17, 1978 and in revised form August 10, 1978. Research supported in part by NSF Grant MCS 77-02208.

University of Alabama

UNIVERSITY, AL 35486 



\section{PACIFIC JOURNAL OF MATHEMATICS}

\section{EDITORS}

RICHARD ARENS (Managing Editor)

University of California

Los Angeles, California 90024

C. W. CURTIS

University of Oregon

Eugene, OR 97403

C. C. MOORE

University of California

Berkeley, CA 94720
J. DugundjI

Department of Mathematics University of Southern Californı Los Angeles, California 90007

R. Finn and J. Milgram Stanford University Stanford, California 94305

ASSOCIATE EDITORS

E. F. BECKENBACH

B. H. NeUmanN

F. WOLF

K. YoSHIDA

\section{SUPPORTING INSTITUTIONS}

UNIVERSITY OF BRITISH COLUMBIA CALIFORNIA INSTITUTE OF TECHNOLOGY

UNIVERSITY OF CALIFORNIA

MONTANA STATE UNIVERSITY

UNIVERSITY OF NEVADA, RENO

NEW MEXICO STATE UNIVERSITY

OREGON STATE UNIVERSITY

UNIVERSITY OF OREGON
UNIVERSITY OF SOUTHERN CALIFORNIA

STANFORD UNIVERSITY

UNIVERSITY OF HAWAII

UNIVERSITY OF TOKYO

UNIVERSITY OF UTAH

WASHINGTON STATE UNIVERSITY

UNIVERSITY OF WASHINGTON 


\section{Pacific Journal of Mathematics}

\section{Vol. 80, No. $1 \quad$ September, 1979}

Jeroen Bruijning and Jun-iti Nagata, A characterization of covering dimension by

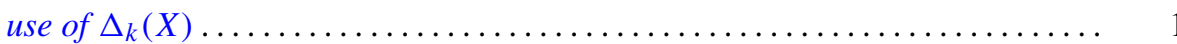

John J. Buoni and Albert Jonathan Klein, On the generalized Calkin algebra ...... 9

Thomas Ashland Chapman, Homotopy conditions which detect simple homotopy

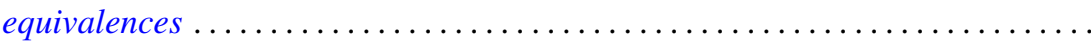

John Albert Chatfield, Solution for an integral equation with continuous interval

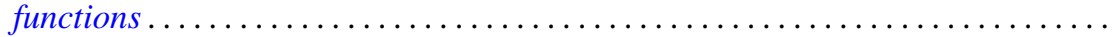

Ajit Kaur Chilana and Ajay Kumar, Spectral synthesis in Segal algebras on

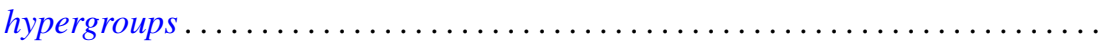

Lung O. Chung, Jiang Luh and Anthony N. Richoux, Derivations and

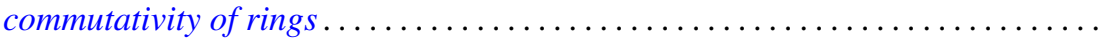

Michael George Cowling and Paul Rodway, Restrictions of certain function spaces to closed subgroups of locally compact groups .....................

David Dixon, The fundamental divisor of normal double points of surfaces........

Hans Georg Feichtinger, Colin C. Graham and Eric Howard Lakien,

Nonfactorization in commutative, weakly selfadjoint Banach algebras . . . . . . .

Michael Freedman, Cancelling 1-handles and some topological imbeddings ....... .

Frank E., III Gerth, The Iwasawa invariant $\mu$ for quadratic fields . . . . . . . . . . . . . .

Maurice Gilmore, Three-dimensional open books constructed from the identity

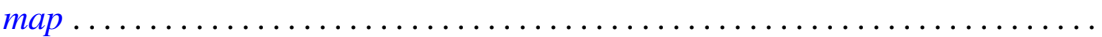

Stanley P. Gudder, A Radon-Nikodým theorem for $*$-algebras .

Peter Wamer Harley, III and George Frank McNulty, When is a point Borel? .

Charles Henry Heiberg, Fourier series with bounded convolution powers . .

Rebecca A. Herb, Characters of averaged discrete series on semisimple real Lie

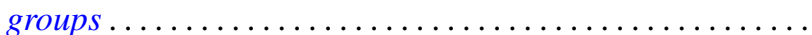

Hideo Imai, On singular indices of rotation free densities . .

Sushil Jajodia, On 2-dimensional CW-complexes with a single 2-cell . . .

Herbert Meyer Kamowitz, Compact operators of the form $u C_{\varphi}$

Matthew Liu and Billy E. Rhoades, Some properties of the Chebyshev method...

213

George Edgar Parker, Semigroups of continuous transformations and generating

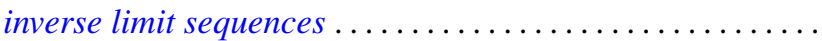

Samuel Murray Rankin, III, Oscillation results for a nonhomogeneous

equation ...

Martin Scharlemann, Transverse Whitehead triangulations ...

Gary Joseph Sherman, A lower bound for the number of conjugacy classes in a

finite nilpotent group

Richard Arthur Shoop, The Lebesgue constants for $\left(f, d_{n}\right)$-summability .

Stuart Jay Sidney, Functions which operate on the real part of a uniform

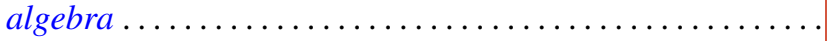

Tim Eden Traynor, The group-valued Lebesgue decomposition 